\title{
A Conversation with Yukiko Yamashita
}

\author{
INTERVIEWER: KIM BAUMANN \\ Chief Editor, Nature Reviews Molecular Cell Biology
}

\begin{abstract}
Yukiko Yamashita is the James Playfair McMurrich Collegiate Professor of the Life Sciences and a Professor in the Department of Cell and Developmental Biology at the University of Michigan Medical School and the University of Michigan Life Sciences Institute.
\end{abstract}

Kim Baumann: The main focus of your laboratory is studying the mechanisms that regulate asymmetric cell division, but you also work on chromocenters. I know they have been described for about a century from a cytological point of view, but could you tell us what they are and how you became interested in them?

Dr. Yamashita: The last question first. We don't get to dictate what to study. As is so often the case, our data lead us in a direction. We pretty much bumped into this. We had been studying asymmetric stem cell division for a very long time. Then four years back, we published one paper about asymmetric stem cell division. For that study, we needed a chromosome-specific repetitive sequence as a probe. When we looked into the probe sequence as a tool, inevitably we noticed these satellite DNAs had very interesting distributions across all those chromosomes. That really led us to look into this satellite DNA, which is a major constituent of pericentromeric heterochromatin. They're repetitive DNA, like very short telomere repeats. That's why it skews ATGC contents. As a result, when you do the centrifugation on the cesium chloride, those DNA settle in a different way compared to the major euchromatic DNA. That's the original reason they were called satellite DNA. These repeats exist mainly in the pericentromeric heterochromatin. This pericentromeric heterochromatin has been known to form chromocenters for, as you said, about a century. Of course, the function of the centromere is very well known and established, but the pericentromeric heterochromatin that excludes the centromeric sequence, as well as the chromocenter that it forms, neither of their functions have been shown.

We decided to look into its potential function and we studied a protein that binds to satellite DNA. Nowadays, you can do pretty much anything with CRISPR-Cas9, but one thing you probably can't do is to "CRISPR out" the entire pericentromeric heterochromatin because that spans ten megabases or more sometimes. What you can do, is you can potentially remove the protein that binds to the chromocenter. We thought that'd be a really good starting point. Luckily for us, once we removed those chromocen- ter-binding proteins, what happens is that the chromocenter becomes dispersed.

Kim Baumann: Are these proteins that bind to satellite DNAs specific to the pericentromeric area, or is it where there are other satellite DNAs?

Dr. Yamashita: The reason we got into this protein was that, probably a few decades back, people had identified it biochemically, starting from a simple repeat sequence to see what proteins might bind to it. Through that, this protein's biochemical function has been known for a really long time. This protein binds to simple AATAT repeat satellites, but nobody really understood the actual cell biological function of that protein. So that's why this time we decided to go into a little bit of detail to look at its phenotype and to try to tie it back to actual cell biological function.

Kim Baumann: What happens if you remove this protein?

Dr. Yamashita: Normally, the chromocenter is the association of pericentromeric heterochromatin from many chromosomes. For example, if you have ten chromosomes in your cell, that could cluster into three. But if you remove this protein, instead of three chromocenters, there can be six or seven. The number increases, suggesting that pericentromeric heterochromatin association is somehow perturbed.

The next thing that happens after you remove the chromocenter protein is that individual chromosomes start floating out of the nucleus to form micronuclei, which was quite a surprise to us.

Kim Baumann: Is this during cell division?

Dr. Yamashita: In interphase. If you take a look at the wild-type nucleus, it is very stable: always round, and it stays that shape. If you perturb the chromocenter formation, all of a sudden this round nucleus starts budding off to spit out a few chromosomes. This really told us that the chromocenter formation is a way to somehow link all those chromosomes into a sort of network.

(C) 2017 Yamashita. This article is distributed under the terms of the Creative Commons Attribution-NonCommercial License, which permits reuse and redistribution, except for commercial purposes, provided that the original author and source are credited. 
Kim Baumann: You think that all cell types have some sort of chromocenter?

Dr. Yamashita: Yes. That is actually a very conserved feature. The chromocenter has been observed in essentially almost all cell types. Plant cells, mammalian cells, Drosophila cells, essentially any.

Kim Baumann: Can you tell us a bit more about micronuclei? My understanding is that they can form at the end of cell division if something was wrong. How do you think that links to your observations?

Dr. Yamashita: We also looked at all the bad things that can happen when micronuclei form. For example, when nuclear envelope integrity is disrupted, DNA damage happens inside. There is similarity, but also some differences, between those lagging-chromosome-induced micronuclei and chromocenter-disruption-induced micronuclei. When you disrupt the chromocenter, it's not just micronuclei; even the major macronuclei have all sorts of problems with nuclear envelope integrity as well as protection from DNA damage.

Kim Baumann: So, there is increased DNA damage in those cases?

Dr. Yamashita: Two pathways lead to this final consequence. One: you have the lagging chromosome that can lead to micronuclei formation and then lead to a nuclear integrity problem. The other way, the nuclear integrity can be heavily disrupted if you don't have a chromocenter, and that can lead to micronuclei formation. That said, there are very interesting parallels: similarities, but a little bit of difference to it.

Kim Baumann: In which cell types have you studied this? Is it across more than one species?

Dr. Yamashita: First, we looked into germ cells in Drosophila testis, simply because that's the system in which we always work. Of course, we wanted to know if there are weird things about our model system, so we expanded this to mouse cell culture. Once you make a probe for a certain pericentromeric satellite, it's easier to go across many different mouse cells instead of going to other species. We took a look at fibroblasts, immune cells, many different kinds of cell types in the mouse, and the phenotype is always very consistent.

Kim Baumann: Do you think this is going to become a major interest for your life? What are the next steps?

Dr. Yamashita: For a while, because not much has been known about the chromocenter or pericentromeric heterochromatin, so there is a lot we can do. I'm looking forward to it. I think we're going to work on many aspects of this pericentromeric heterochromatin in the coming years. But at the same time, I think what we try in the laboratory is that everybody really takes on a different project so that when they become independent, not only are they independent of me but independent of each other. That said, if we extend from the pericentromeric heterochromatin, I'm looking into the way that it's going to be very discrete from what we discover here, now.

Kim Baumann: In terms of satellite DNAs, do you have any other lines of research that are related to these repetitive sequences across the genome?

Dr. Yamashita: We are finally convinced that pericentromeric heterochromatin has an important function. It was believed for a very long time to be junk. Not many people really cared how pericentromeric heterochromatin was taken care of by the host cells or actual cells, because they were believed to be junk or parasites: selfish DNA elements.

Kim Baumann: But these areas are very conserved, right?

Dr. Yamashita: It's conserved in terms of the characteristics, but if you look at the sequence, it's very different across species. I believe that was one reason that, for a long time, people didn't really appreciate that pericentromeric heterochromatin has such a fundamental biological function. Now that we know it has a function, I'm very interested how this vast array of the pericentromeric heterochromatin is maintained. This is probably a similar question to telomere maintenance. That has been studied back when Liz Blackburn and Carol Greider started to look into how this is really maintained, what kind of enzyme was doing it.

Kim Baumann: You mean maintain in terms of length?

Dr. Yamashita: Yes. The actual biochemical problem would be different, because pericentromeric satellite DNA is in the middle of the chromosome, so you don't have to worry about the end-replication problem, but how to maintain a repeat: that's conceptually similar. I believe there has to be some mechanism to maintain it. That's one thing I'm very keen on doing in the near future.

Kim Baumann: Now that you've discovered that it plays such an important function, do you think there will be more studies on the chromocenter?

Dr. Yamashita: You mean, from other people? That, I very much look forward to. Of course, that may get into competition. But if you're afraid of competition that means you'll be making discoveries all by yourself. That will be slow. I would actually look forward to more people joining this endeavor. 


\section{$\$_{\text {CSH\& }}^{\infty}$ Cold Spring Harbor Symposia SYMPOSIA}

\section{A Conversation with Yukiko Yamashita}

Cold Spring Harb Symp Quant Biol 2017 82: 416-417 originally published online April 26, 2018 Access the most recent version at doi:10.1101/sqb.2017.82.034983

Creative This article is distributed under the terms of the

Commons http://creativecommons.org/licenses/by-nc/4.0/, which permits reuse and

License redistribution, except for commercial purposes, provided that the original author and source are credited.

Email Alerting Receive free email alerts when new articles cite this article - sign up in Service the box at the top right corner of the article or click here. 